\title{
William W. FORTENBAUGH \& Stephen A. White (éd.), Aristo of Ceos. Text, Translation, and Discussion
}

Transaction Publishers, New Brunswick (U.S.A.)-Londres, 2006 (Rutgers

University Studies in Classical Humanities, 13), $x+373 p$

\section{David Lefebvre}

\section{(2) OpenEdition}

Journals

\section{Édition électronique}

URL : https://journals.openedition.org/philosant/2400

DOI : 10.4000/philosant.2400

ISSN : 2648-2789

Éditeur

Éditions Vrin

\section{Édition imprimée}

Date de publication : 30 octobre 2010

Pagination : 287-290

ISBN : 978-2-7574-0179-8

ISSN : 1634-4561

Référence électronique

David Lefebvre, « William W. Fortenbaugh \& Stephen A. white (éd.), Aristo of Ceos. Text, Translation, and Discussion », Philosophie antique [En ligne], 10 | 2010, mis en ligne le 11 juillet 2019, consulté le 09 décembre 2022. URL : http://journals.openedition.org/philosant/2400 ; DOI : https://doi.org/10.4000/ philosant.2400

\section{(c) (i) $\odot$}

Creative Commons - Attribution - Pas d'Utilisation Commerciale - Pas de Modification 4.0 International - CC BY-NC-ND 4.0

https://creativecommons.org/licenses/by-nc-nd/4.0/ 
est celui de la «localisation» de l'activité, S. Broadie met également au jour le caractère anti-platonicien de l'affirmation suivant laquelle les activités transitives (soit les mouvements) sont plus de l'ordre de la fin que la puissance. Cette affirmation vise selon elle à poser la positivité ontologique du mouvement et des individus et leur indépendance par rapport à toute forme d'Idée. Enfin, R. Chiaradonna étudie la façon dont, en Ennéades, VI, 1, 42, Plotin reprend et modifie la conception aristotélicienne du mouvement. L'erreur d'Aristote serait selon Plotin d'avoir confondu deux plans hétérogènes du mouvement: le plan empirique et « cinématique » (p. 484) du mouvement qui se caractérise bien par une incomplétude; et le plan causal et «dynamique » (p. 484) qui correspond au contraire à l'energeia du mouvement et qui se rattache in fine à l'âme comme "principe dynamique du monde naturel» (p. 484).

L'ouvrage offre ainsi une assez large perspective sur les usages et les enjeux liés à l'histoire de la notion de dynamis ainsi que sur la façon dont Aristote a hérité d'une tradition qu'il a radicalement reconfigurée en connectant la dynamis à l'energeia. La remarquable qualité des contributions et de l'ensemble semble par ailleurs tenir à l'unité des problèmes traités et au dialogue, implicite ou explicite, que celle-ci établit ainsi entre les auteurs. Notons enfin que chaque article comporte une notice bibliographique et que l'ouvrage est complété par un index locorum.

\section{Haud GUÉGUEN-PORCHER}

William W. Fortenbaugh \& Stephen A. White (éd.), Aristo of Ceos. Text, Translation, and Discussion. Transaction Publishers, New Brunswick (U.S.A.)Londres, 2006 (Rutgers University Studies in Classical Humanities, 13), x + 373 p.

L'Ariston dont il sera ici question est le péripatéticien né à Ioulis, dans l'Ile de Céos ; il vécut dans la seconde moitié du $\mathrm{III}^{\mathrm{e}}$ siècle av. J.-C., et fut selon toute vraisemblance le successeur de Lycon de Troie, soit le quatrième successeur d'Aristote à la tête du Péripatos ${ }^{1}$. Comme on le sait, il est difficile de faire le départ entre les textes qui lui reviennent (au moins des Erotika Homoia, un Lycon, et peut-être, selon Diogène Laërce, $V, 64$, un recueil de vies de philosophes, en particulier du Lycée, avec leurs testaments, utilisés par Diogène) et ceux attribuables à son homonyme, le stoïcien Ariston de Chios. Cette nouvelle édition des fragments et principalement des témoignages recueillis à son sujet est appelée à compléter très utilement les éditions de Knögel (Der Peripatetiker Ariston von Keos bei Philodem, Leipzig, 1933, p. 79-94) et surtout de Wehrli (Die Schule des Aristoteles, VI, Lycon und Ariston von Keos, Bâle-Stuttgart, 1968, p. 32-44). Elle prend place dans la série des RUSCH en cours de publication qui se propose d'actualiser l'édition classique de l'école d'Aristote établie par Wehrli, en adjoi-

1. Vita menagiana, ligne 20 Düring $=4 \mathrm{~A}$ SFOD et, dans le même sens, les témoignages de Cicéron et Plutarque ( 9 et 3 SFOD). Par rapport à Wehrli, SFOD ajoutent à ce sujet un texte des Stromates (I, XIV, 63, 5) où Clément ne mentionne justement pas Ariston dans la succession d'Aristote, contrairement à ce qu'on lit dans le $D P h A, \mathrm{I}, \mathrm{p} .398$. 
gnant aux textes commentés une traduction en langue anglaise et des études ${ }^{2}$. On trouve donc ici, réalisées par Peter Stork, Tiziano Dorandi, William W. Fortenbaugh et Johannes M. van Ophuijsen (en abrégé SFOD ; Stork est aussi parfois présenté comme le seul responsable de l'édition), une nouvelle présentation annotée des témoignages ou fragments, avec davantage de contexte, et une traduction. Les textes sont classés en cinq rubriques (Vie, Écrits, Disputés, Dits, Non acceptés). Fait suite un Index des citations du nom "Ariston» (selon qu'il est cité seul ou avec une épithète, " originaire de Ioulis », «de Céos» ou le "philosophe péripatéticien»), une concordance avec Wehrli, Knögel et les éditions des fragments de l'homonyme stoïcien (telles que les $S V F$ et le livre de A.M. Ioppolo sur Aristone di Chio e lo Stoicismo Antico, 1980) et plusieurs Indices. Il ne s'agit pas à proprement parler d'une nouvelle édition de tous les textes mais d'une actualisation de la présentation des textes attribués à Ariston à partir des meilleures éditions disponibles des auteurs qui le citent, éditions d'ailleurs corrigées le cas échéant ${ }^{3}$. L'apport du volume réside en particulier dans l'édition partiellement nouvelle des textes pertinents du papyrus de Philodème et dans leur traduction, la première en langue anglaise (p. 219, n. 7). Par rapport à Wehrli, les choix éditoriaux, ici comme pour les autres volumes de la même collection, sont moins exclusifs, ce qui permet à SFOD d'introduire 22 textes nouveaux. Même si la plus grande partie (17) sont relégués dans la rubrique des textes "non acceptés» ou ne mentionnent pas le nom d'Ariston, l'ensemble recueille de manière plus complète et plus ouverte le matériel disponible sur Ariston ou plutôt les Ariston. On notera en particulier l'introduction dans ce recueil d'un fragment, inconnu de Wehrli, extrait du $\Pi \varepsilon \rho i$

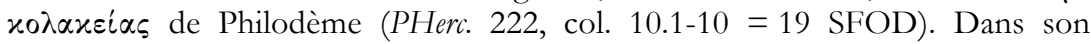
étude, Dorandi (p. 229) n'exclut pas de voir dans l'Ariston qui y est mentionné le péripatéticien et considère comme plausible l'hypothèse que Philodème se réfère dans ce texte comme dans le fragment 20 SFOD issu du même traité

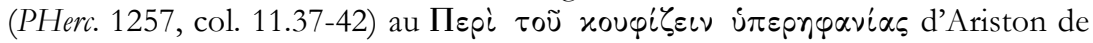
Céos.

La seconde partie du livre (p. 179-359) réunit huit études. Dans la première, «In search of Aristo of Ceos », David Hahm, pour sortir du problème selon lui exclusivement «moderne » de la distinction entre les deux Ariston, donne une présentation chronologique de la réception des deux auteurs selon les différentes traditions de témoignage (la mention du testament de Straton dans Diogène Laërce, Panétius, Cicéron et Strabon, enfin Plutarque, Athénée et Stobée). Il en sort une histoire de la perception d'Ariston, d'abord comme auteur de biogra-

2. IX : Démétrios de Phalère ; X : Dicéarque de Messine ; XI : Eudème de Rhodes ; XII : Lycon de Troie et Hiéronymos de Rhodes (voir Philosophie Antique, 6, 2006, p. 213-216). Les prochains volumes à paraitre portent sur Héraclide du Pont et Straton de Lampsaque.

3. Diogène Laërce est cité d'après l'édition de Marcovich (Teubner, 1999-2002) confirmée par les collations de T. Dorandi pour sa nouvelle édition des Vies (p. 5-6) et le livre X de Philodème, De Vitiis, est cité d'après l'édition de Jensen (Teubner, 1911) mais SFOD ont eu accès à la reconstruction inédite de la colonne 10 du PHerc. 1008 avec des lectures nouvelles dues à Anna Angeli et Mario Capasso. Une nouvelle édition due à Giovanni Indelli est annoncée (voir dans l'étude de T. Dorandi, p. 219). 
phies ou de successions de philosophes, mais surtout l'idée que la confusion des Ariston est un problème moderne et non antique, qui s'est nourri de la remarque de Panétius rapportée par Diogène (VII $163=8$ SFOD) selon laquelle la plupart des œuvres d'Ariston de Chios (sauf les Lettres) seraient en réalité de l'Ariston péripatéticien. La stratégie de Panétius de purger la tradition stoïcienne des œuvres d'Ariston de Chios coupable de déviationnisme cynique aurait semé le trouble dans la constitution du corpus de l'homonyme aristotélicien. L'étude de Hahm qui donne une intéressante présentation des témoignages laisse cependant entièrement de côté le problème de l'interprétation du matériel conservé sur papyrus, pour lequel la question de l'attribution se pose au premier chef. Les quatre études suivantes abordent justement cette question très disputée de l'origine du plus long et du plus intéressant des textes édités, un extrait d'un

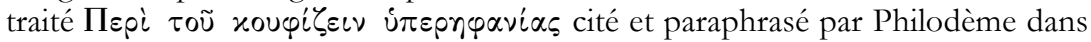
son De vitiis (PHerc 1008, col. 10.10-30;21a-21o SFOD et 13,1-7 Wehrli) : «I frammenti papiracei di Aristone di Ceo » (Tiziano Dorandi) ; «L'autore del Пєрi

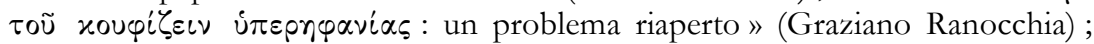
«Characters in Aristo » (Sabine Vogt) et «Aristotle on Blends of Arrogance » (Voula Tsouna). Seul dans ce recueil, Ranocchia défend la thèse de l'attribution de ces textes à l'Ariston stoïcien, comme l'avait déjà fait notamment Ioppolo (1996), ce qui conduit raisonnablement SFOD à reléguer le traité dans la rubrique des textes d'attribution «disputée». Dorandi fait l'état de la question de l'identification de l'Ariston cité par Philodème depuis la première édition (Caterino, 1827) ${ }^{4}$ et, à partir du texte de Jensen corrigé par Anna Angeli, donne, contre Ranocchia, des éléments nouveaux en faveur de l'attribution de ces fragments à Ariston de Céos. Ranocchia ${ }^{5}$ maintient de son côté l'hypothèse de

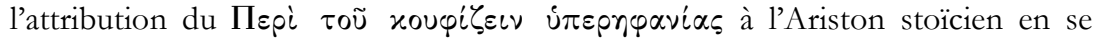
basant notamment sur la nature épistolaire du texte cité par Philodème (l'un des enjeux de la reconstruction de la colonne 10 du PHerc. 1008 est la lecture des lignes 12-13, où Philodème indique la nature du texte qu'il cite : une lettre, pour Dorandi et Ranocchia notamment, un epitome, pour Jensen et Wehrli). L'examen des manuscrits n'est pas le seul recours pour éclairer cette question et Ranocchia introduit dans la suite de son étude (p. 251-257) des éléments issus cette fois de

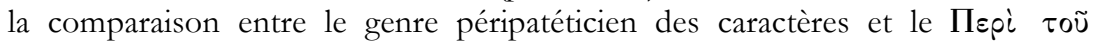

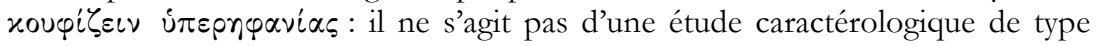
péripatéticien mais les éléments descriptifs sont au service d'un projet parénétique visant, comme l’indique le titre, à «libérer» le destinataire de cette lettre protreptique de son arrogance - cette finalité exhortative étant pour l'auteur bien sûr un élément supplémentaire à l'appui d'une attribution au stoïcien. C'est justement la question du genre philosophique du texte que discutent Sabine Vogt et Voula Tsouna, qui assument l'attribution péripaté-

4. Sur le status quaestionis, voir aussi des éléments dans la contribution de V. Tsouna, note 1, p. $279-280$.

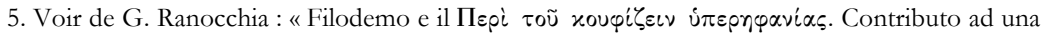
nuova edizione del PHerc. 1008 », Papyrologica Lupiensia, 10 (2001), p. 231-263, et Aristone : Sul modo di liberare dalla superbia, nel decimo libro De vitiis di Filodemo, Florence, Olschki, 2007. 
ticienne de ces textes. De manière utile et très intéressante, Sabine Vogt (éditrice

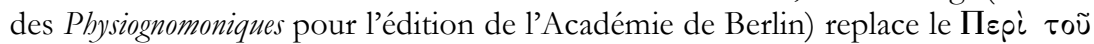

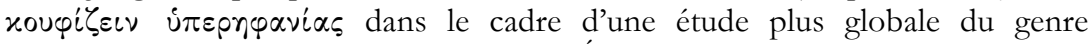
péripatéticien des caractères (Rhétorique et Éthiques d'Aristote, Des vertus et des vices et les Physiognomoniques du pseudo-Aristote, et les Caractères de Théophraste). À partir d'une analyse de la méthode ("inférentielle» ou «conceptuelle») de caractérisation et d'une comparaison de la description des mêmes traits de caractère, elle conclut que l'auteur du traité sur l'arrogance associe les deux voies et peut être identifié au péripatéticien. Dans une lecture interne très fine des mêmes textes, Voula Tsouna montre de quelle façon Ariston conçoit l'arrogance comme un mélange structuré de dispositions, comme un vice «focal» capable de réunir autour de lui d'autres traits psychologiques et éthiques. La contribution de Denis M. Searby, "Aristo of Ceos in the Greek Gnomologies» examine l'absence de dicta attribués à Ariston de Céos dans la tradition gnomologique grecque et penche pour l'attribution au stoïcien de ceux attribués simplement à «Ariston» ou à «Ariston le philosophe ». Les deux études finales, "Natural Philosophy in the Peripatos after Strato ( Robert W. Sharples) et «Peripatetic Biology and the Epitome of Aristophanes of Byzantium» (Oliver Hellmann) complètent heureusement le volume sans entretenir pour autant de rapport direct avec Ariston. L'une et l'autre abordent la question de la postérité des études physiques d'Aristote, Robert Sharples au sein du Péripatos, Oliver Hellmann dans l'Epitome d'Aristophane de Byzance. Ils aboutissent à des conclusions qui ne sont pas sans rapport. Pour R. Sharples, les très rares traces d'une activité dans le domaine de la philosophie de la nature chez Lycon, Hiéronymos et Ariston doivent être prises avec précaution dans la mesure où rien ne garantit qu'elles procédaient d'un intérêt réel de ces philosophes pour l'étude de la nature. Si l'on trouve chez Critolaos, le successeur d'Ariston, l'expression d'un certain nombre de thèses physiques typiquement aristotéliciennes, il faut peut-être moins y voir les signes d'un retour aux études physiques que ceux d'un besoin de manifester la différence et l'originalité du Péripatos vis-à-vis des thèses des écoles rivales, dont notamment le stoïcisme. Hellmann, au terme d'une lecture assez singulière de l'Epitome, en conclut que son objet véritable n'était pas la zoologie mais que sa fonction première devait être de fournir une sorte de lexique à l'usage de poètes savants «à la recherche d'un matériel pour décrire un animal dans une œuvre poétique mais sans intérêt pour la biologie scientifique » (p. 354). L'ensemble du volume, où l'on rencontre ici et là quelques erreurs matérielles inattendues, constitue un instrument sans aucun doute très précieux pour la connaissance de l'histoire du Péripatos et de la tradition du genre des caractères, au-delà pour l'histoire philosophique des vices.

David LEFEBVRE

Angelo GiavatTo, Interlocutore di se stesso: la dialettica di Marco Aurelio, Hildesheim, Olms, 2008 (Europaea memoria. Studien, 58), VIII + 270 p. ISBN 9783487136417

L’ouvrage proposé par Angelo Giavatto (AG) est la version revue et corrigée d'une thèse de doctorat en philologie grecque et latine, soutenue à l'Université 